\title{
Second harmonic generation spectroscopy on hybrid plasmonic/dielectric nanoantennas
}

\author{
Heiko Linnenbank ${ }^{1}$, Yevgen Grynko², Jens Förstner ${ }^{2}$ and Stefan Linden ${ }^{1}$
}

Plasmonic nanoantennas provide unprecedented opportunities to concentrate light fields in subwavelength-sized volumes. By placing a nonlinear dielectric nanoparticle in such a hot spot, one can hope to take advantage of both the field enhancement provided by nanoantennas and the large, nonlinear optical susceptibility of dielectric nanoparticles. To test this concept, we combine gold gap nanoantennas with second-order, nonlinear zinc sulfide nanoparticles, and perform second harmonic generation (SHG) spectroscopy on the combined hybrid dielectric/plasmonic nanoantennas as well as on the individual constituents. We find that SHG from the bare gold nanoantennas, even though it should be forbidden due to symmetry reasons, is several orders of magnitude larger than that of the bare zinc sulfide nanoparticles. Even stronger second harmonic signals are generated by the hybrid dielectric/plasmonic nanoantennas. Control experiments with nanoantennas containing linear lanthanum fluoride nanoparticles reveal; however, that the increased SHG efficiency of the hybrid dielectric/plasmonic nanoantennas does not depend on the nonlinear optical susceptibility of the dielectric nanoparticles but is an effect of the modification of the dielectric environment. The combination of a hybrid dielectric/plasmonic nanoantenna, which is only resonant for the incoming pump light field, with a second nanoantenna, which is resonant for the generated second harmonic light, allows for a further increase in the efficiency of SHG. As the second nanoantenna mediates the coupling of the second harmonic light to the far field, this double-resonant approach also provides us with control over the polarization of the generated light.

Light: Science \& Applications (2016) 5, e16013; doi:10.1038/Isa.2016.13; published online 15 January 2016

Keywords: nanoantennas; plasmonics; second harmonic generation

\section{INTRODUCTION}

Since the pioneering experiments of $\mathrm{H}$. Hertz and G. Marconi, antennas have been essential tools for the manipulation of electromagnetic waves $^{1,2}$. Thus far, antennas have been mainly used for radio and microwave applications, e.g., as components of wireless communication systems or for object detection and ranging ${ }^{3}$. The development of high precision nano-fabrication techniques has additionally revealed promising possibilities for antennas in the optical domain. For instance, one can nowadays fabricate nanometer-sized metallic nanostructures that exhibit resonances in the near infrared and even in the visible spectral regime ${ }^{4-6}$. Like their counterparts in the radio or microwave regime, these so-called plasmonic nanoantennas can be used to funnel-free propagating waves into volumes far smaller than the diffraction limit and vice versa ${ }^{4-7}$. This results in an enormous intensity enhancement for a resonant incoming light field and provides an enhanced far-field coupling for nanoscale light sources.

One class of optical processes with a strong demand for high intensities is the class of nonlinear optical frequency conversion processes ${ }^{5,8}$. The most prominent among them are second harmonic generation (SHG) and parametric down-conversion in noncentrosymmetric bulk crystals (noncentrosymmetry is a necessary condition for electric dipole allowed second-order nonlinear optical processes, see, e.g., Boyd $^{8}$, which are both widely used for the generation of high power, coherent, tunable radiation, from the ultraviolet up to the mid-infrared range ${ }^{9-11}$. Through an increased demand for subwavelength coherent light sources, an interest in subwavelength frequency conversion schemes has also emerged ${ }^{12-19}$. A simple approach could be to directly use nanometer-sized nonlinear dielectric crystals for frequency conversion applications. However, the efficiency of these processes is a superlinear function of the volume of the employed crystals ${ }^{20}$. Therefore, nonlinear frequency conversion processes based on such nonlinear dielectric nanocrystals are intrinsically very weak.

A strategy to overcome the poor conversion efficiency of nonlinear dielectric nanocrystals could be to place the nanocrystals in the hot spots of plasmonic nanoantennas so that one can take advantage of both the plasmonic field-enhancement effect and the large nonlinear optical susceptibility of the dielectric nanoparticles. In several experiments on $\mathrm{SHG}^{21-23}$ and third harmonic generation (THG) ${ }^{24,25}$ from such hybrid plasmonic/dielectric nanoantennas, an enhancement of the nonlinear efficiency of the hybrid dielectric/plasmonic nanoantennas compared to that of the corresponding bare, nonlinear dielectric nanocrystals was indeed observed. These findings apparently support the idea behind the aforementioned strategy, i.e., the increase in the nonlinear signal is caused by a combination of plasmonic field enhancement and a large dielectric nonlinear susceptibility. However, a recent experimental study on THG from gap nanoantennas containing 
indium tin oxide (ITO) nanoparticles casts serious doubts on this interpretation ${ }^{25}$. By performing THG spectroscopy on different sized gap nanoantennas, Metzger et al. found strong evidence that the nonlinear enhancement of the investigated hybrid plasmonic/ dielectric nanoantennas is mainly related to changes in the linear optical properties of the gap nanoantenna resonances due to the presence of the ITO nanoparticles and not the third-order nonlinearity of the nanoparticles. Furthermore, they identified the gold nanoantennas as the dominant source of the THG emission.

Plasmonic nanoantennas can be used not only to concentrate the incident pump field in small volumes at the position of the nonlinear nanocrystal but also to help control the coupling of the generated
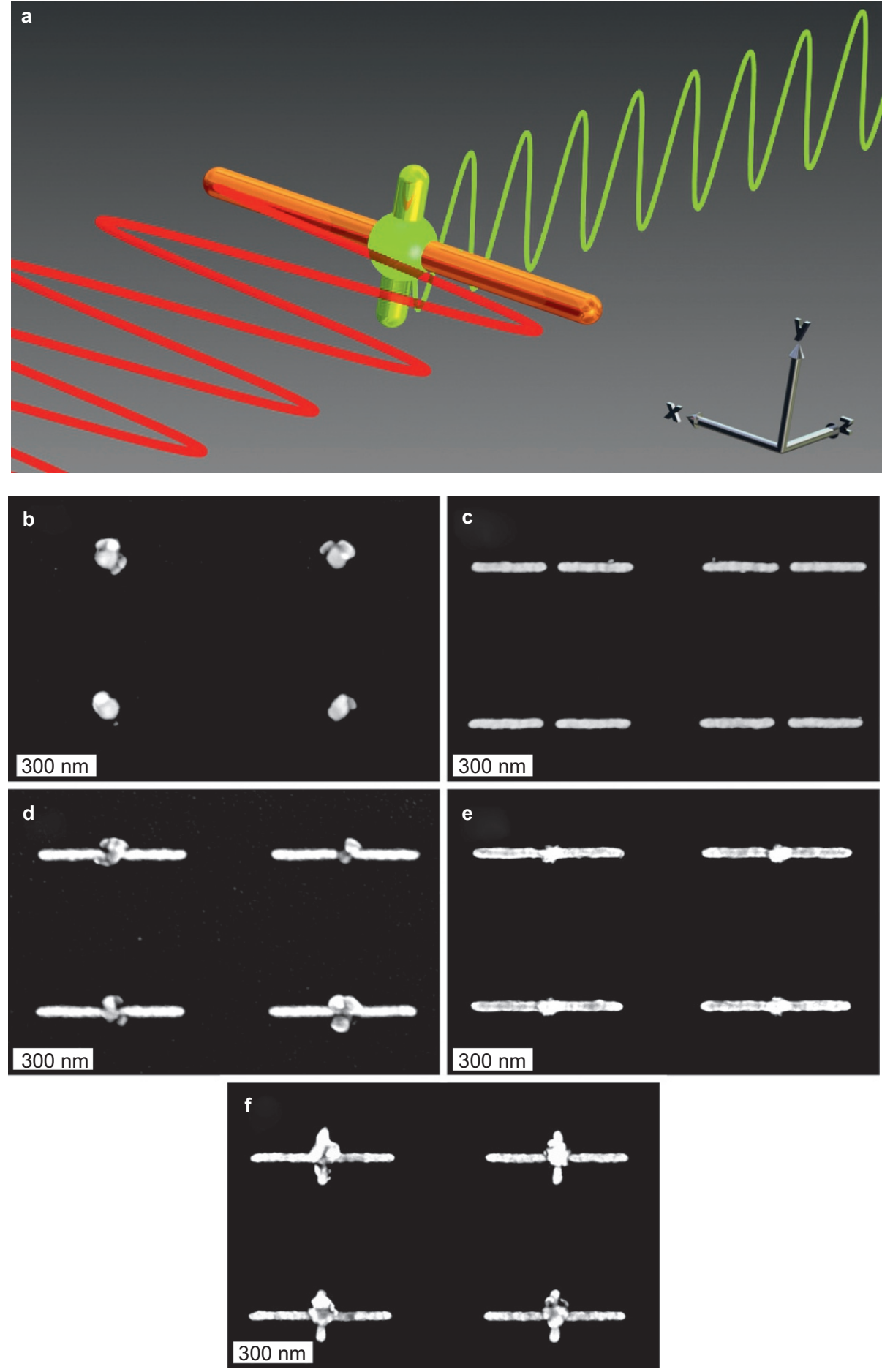

Figure 1 (a) Schematic sketch of a double-resonant hybrid plasmonic/dielectric nanoantenna system together with the coordinate system used in this work. (b)-(f) Top-view SEM micrographs of the investigated $\mathrm{ZnS}$ nanostructures, bare gold nanoantennas, nanoantennas with $\mathrm{ZnS}$ nanoparticles, nanoantennas with LaF 3 nanoparticles, and double-resonant nanoantenna systems with ZnS nanoparticles. 
second harmonic (SH) light to the far field. To combine both effects in a single nanostructure, a plasmonic nanoantenna must be designed that it is resonant for both the incoming pump light field and the locally generated $\mathrm{SH}$ light field ${ }^{26-28}$. This can, for example, be done by combining two differently sized plasmonic nanoantennas and a nonlinear dielectric nanoparticle to create a double-resonant hybrid plasmonic/dielectric nanoantenna system, as shown in Figure la. In a classical resonator picture, this geometry can be seen as a miniaturized version of a double-resonant cavity used for intracavity SHG, which is known to show higher conversion efficiencies than the case in which only the pump light or the SH light is resonantly enhanced $^{29-32}$.

In this work, we study the origin of the SHG enhancement in hybrid plasmonic/dielectric nanoantennas. For this purpose, we combine gap nanoantennas made of gold either with nonlinear zinc sulfide nanoparticles or linear (in this context, linear means that the second-order nonlinear susceptibility vanishes due to symmetry reasons) lanthanum fluoride nanoparticles and perform linear extinction measurements and SHG spectroscopy on the hybrid dielectric/ plasmonic nanoantennas, as well as on the individual constituents. We find that the SHG efficiency of the bare gold nanoantennas is several orders of magnitude higher than that of the zinc sulfide nanoparticles. A comparison of the SHG efficiency of the two types of hybrid nanoantennas shows that this process is very sensitive to changes of the environment of the nanoantennas, but independent of the nonlinear properties of the dielectric nanoparticles. Additionally, we combine two plasmonic nanoantennas such that one nanoantenna is resonant for the pump field, while the second nanoantenna is resonant for the generated $\mathrm{SH}$ light. With this approach, we can not only achieve a higher conversion efficiency but also control the polarization state of the generated SH light.

\section{MATERIALS AND METHODS}

The preparation of the nanoantennas was done by a two-step electron beam lithography process. In the first electron beam lithography step, we fabricated several arrays of gold nanoantennas and additional alignment marks. In the second lithography step, prior to the exposure, the positions of those marks were detected to determine the positions of the nanoantennas. Subsequently, the electron beam resist was selectively exposed at the regions of the gaps of the antennas. After development, we deposited a $60-\mathrm{nm}$ thin dielectric layer via high vacuum thermal evaporation and performed a lift-off process. For one sample (sample 1), zinc sulfide $(\mathrm{ZnS})$, which is a wide band gap noncentrosymmetric semiconductor, was used as a dielectric, whereas for a second sample (sample 2), lanthanum fluoride $\left(\mathrm{LaF}_{3}\right)$, which has a comparable refractive index to $\mathrm{ZnS}$ but a centrosymmetric crystal structure, was used instead. For our experiments, we chose a gap nanoantenna design, which is known to exhibit only a weak intrinsic SHG efficiency ${ }^{33-35}$. The nanoantennas had a width and a height of 40 $\mathrm{nm}$, a gap width of $50 \mathrm{~nm}$, and were arranged on a $900 \mathrm{~nm} \times 600 \mathrm{~nm}$ lattice with a size of $40 \mu \mathrm{m} \times 40 \mu \mathrm{m}$. The lengths of the nanoantennas (approximately $300 \mathrm{~nm}$ per antenna arm) were chosen such that the linear extinction has a maximum at approximately $1500 \mathrm{~nm}$ wavelength. Additionally, dielectric nanoparticles were arranged on a 900 $\mathrm{nm} \times 600 \mathrm{~nm}$ lattice without antennas but with a comparable size to those located in the nanoantenna gaps, and thin dielectric films were fabricated in the second lithography step. In Figure 1, SEM micrographs of the investigated (b) ZnS nanostructures, (c) bare gold nanoantennas, (d) nanoantennas with $\mathrm{ZnS}$ nanoparticles, (e) nanoantennas with $\mathrm{LaF}_{3}$ nanoparticles, and (f) double-resonant nanoantenna systems with ZnS nanoparticles are shown. The SEM micrographs indicate that the $\mathrm{ZnS}$ is in a crystalline state.

The linear response of the nanoantenna arrays was measured with polarization selective, normal-incidence, white-light transmission spectroscopy over a wavelength range from $600 \mathrm{~nm}$ to $1700 \mathrm{~nm}$. For the SHG spectroscopy measurements, we employed a double-pass optical parametric generator (OPG), delivering pulses with a length of 200-300 fs (spectral full width at half maximum 8-40 nm) at 42 $\mathrm{MHz}$ repetition rate, tunable from $1400 \mathrm{~nm}$ to $1650 \mathrm{~nm}$, as pump light source $^{36}$. The average power of the pump beam was attenuated to below $10 \mathrm{~mW}$. Above this value, the nonlinear response of the nanoantenna arrays would begin to drop with time, indicating an opticalinduced thermal damage. The linear polarization in the $x$-direction (see coordinate system in Figure 1a) of the pump beam was controlled by the combination of a polarizer and a half-wave plate. The pump light was focused down to an approximately $30-\mu \mathrm{m}$ spot diameter under normal incidence ( $z$-direction). The SHG signal was recorded in transmission with a charge-coupled device spectrometer for different pump wavelengths in steps of approximately $10 \mathrm{~nm}$. Additionally, a polarizer could be used to characterize the polarization of the $\mathrm{SH}$ light. If not stated otherwise, the SHG signal was recorded polarization unselective. To eliminate any parasitic effects due to changes in the pulse shape and beam divergence when tuning the OPG, the SHG signals from the nanoantenna and nanoparticle arrays were spectrally integrated and referenced to the weak SHG signal obtained from the ITO-covered substrate surface. A dielectric OD 4 longpass filter in front of the focusing lens was used to block any parasitic-generated $\mathrm{SH}$ light from the OPG or used optics. Furthermore, to make sure that the recorded SHG signal indeed stemmed from the investigated structures, we recorded after each measurement spectra without any sample and also with a glass substrate without ITO under the same conditions as those used in the actual measurement.

\section{RESULTS AND DISCUSSION}

To first assess the nonlinear properties of the thermal evaporated dielectrics, we compared the SHG efficiency of the nanoparticle arrays and thin films made from $\mathrm{ZnS}$ and $\mathrm{LaF}_{3}$ with the SHG efficiency of the underlying ITO-covered substrate surface at a fixed wavelength of $1480 \mathrm{~nm}$ (see Figure 2). In the case of the $\mathrm{LaF}_{3}$ nanoparticle arrays/ thin films, the SHG efficiency was weaker than that of the underlying

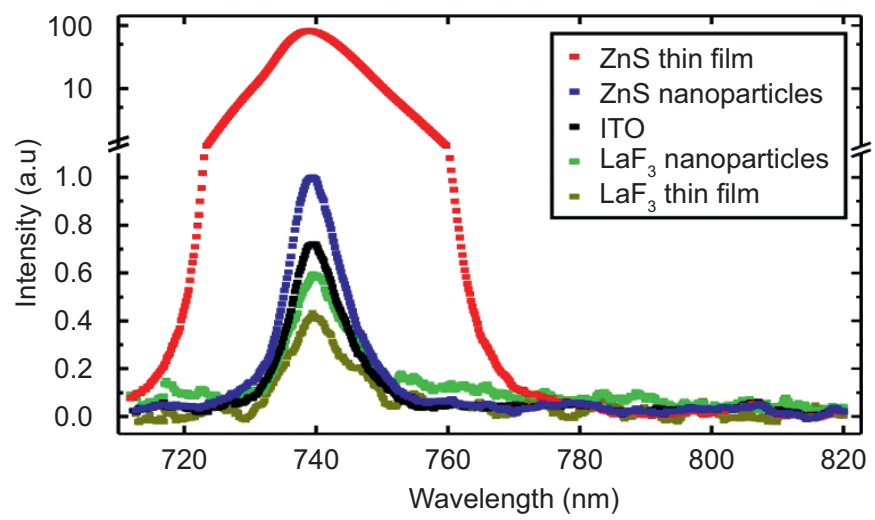

Figure 2 SHG signals obtained, with a fixed pump wavelength of $1480 \mathrm{~nm}$ and constant pump intensity, from a thin film of ZnS (red), a ZnS nanoparticle array (blue), a $\mathrm{LaF}_{3}$ nanoparticle array (green), and a thin film of $\mathrm{LaF}_{3}$ (dark yellow). All nanoparticle arrays and thin films were deposited on a glass substrate covered with a thin layer of ITO. The SHG signal from the ITO-covered substrate surface is shown in black. 
ITO surface. The most likely reason for this observation is scattering/ reflection of the incoming pump light. In the case of the $\mathrm{ZnS}$ nanoparticle arrays/thin films, the SHG efficiency was larger by a factor of $1.4 / 115$ than that of the underlying ITO surface. Additionally, the ratio between the SHG efficiency of the $\mathrm{ZnS}$ nanoparticle arrays and the thin films is in excellent agreement with the theories for random quasi phase matching, taking their volumes into account ${ }^{37-40}$. Therefore, this preliminary experiment indicates that the $\mathrm{ZnS}$ nanoparticles indeed show a second-order nonlinear response, whereas no second-order nonlinear effects can be observed from the $\mathrm{LaF}_{3}$ nanoparticles, as expected.

Next, we compared the SHG efficiency of a nanoantenna array with $\mathrm{ZnS}$ nanoparticles with that of an array of bare $\mathrm{ZnS}$ nanoparticles (see Figure $1 \mathrm{~b}$ and $1 \mathrm{~d}$ ) at the center wavelength of the plasmonic resonance of the nanoantennas. Here, we found that the SHG signal of the hybrid plasmonic/dielectric nanoantenna array was larger than that of the $\mathrm{ZnS}$ nanoparticle array by a factor of 500000 .

To determine the main source of the nonlinear signal of the hybrid plasmonic/dielectric nanoantennas, we compared the linear and nonlinear spectra of an array of bare gold nanoantennas with that of an array of nanoantennas with $\mathrm{ZnS}$ nanoparticles, both located on sample 1. The incorporation of a high refractive index material in the gap of a gold nanoantenna usually leads to a red-shift of the plasmonic resonance $^{25}$, which would prevent a direct comparison. To compensate this red-shift, i.e., to obtain congruent plasmonic resonances, we have chosen hybrid nanoantennas, which are approximately 10\% shorter than the bare gold nanoantennas, for the comparison. The corresponding extinction (one minus measured transmission) spectra for $\mathrm{x}$-polarized normal incident light are shown as continuous lines in Figure 3a. The connected symbols represent the measured SHG spectra, normalized to the maximum SHG signal of the bare gold nanoantennas. For both antenna arrays, the SHG efficiency closely followed the linear extinction, showing that the SHG was indeed governed by the plasmonic resonance of the nanoantennas. A comparison of the SHG efficiencies of both antenna arrays shows a doubling of the SHG efficiency of the nanoantennas due to the incorporation of the $\mathrm{ZnS}$ nanoparticles.

To test whether the increased SHG efficiency really stems from the nonlinear properties of the $\mathrm{ZnS}$, we compared an array of bare gold nanoantennas and a nanoantenna array with $\mathrm{LaF}_{3}$ nanoparticles, both located on sample 2, in the same way as before (see Figure $3 \mathrm{~b}$ ). Here, we again observe a doubling of the SHG efficiency due to the incorporation of a dielectric.

A survey of the presented experimental results allows us to draw several conclusions: (i) Because we obtained the same efficiency enhancement regardless whether we used linear $\mathrm{LaF}_{3}$ or nonlinear $\mathrm{ZnS}$ nanoparticles, we conclude that the SHG efficiency enhancement of the plasmonic nanoantennas due to the incorporation of dielectric nanoparticles does not originate from an interplay between the plasmonic field-enhancement effect and the nonlinear optical properties of the dielectric nanoparticle. (ii) The gold nanoantennas are the dominant source of the SH signal, as the $\mathrm{LaF}_{3}$ nanoparticles show no measurable second-order nonlinearity. (iii) The SHG efficiency of plasmonic nanoantennas can be increased by a modification of the dielectric environment. We attribute this effect to a small but measurable narrowing of the resonance line width of the hybrid nanoantennas relative to the bare gold nanoantennas. This is synonymous with an increase of the quality factor $Q$, which has a strong effect on the nonlinear performance of plasmonic nanostructures ${ }^{25,41}$.
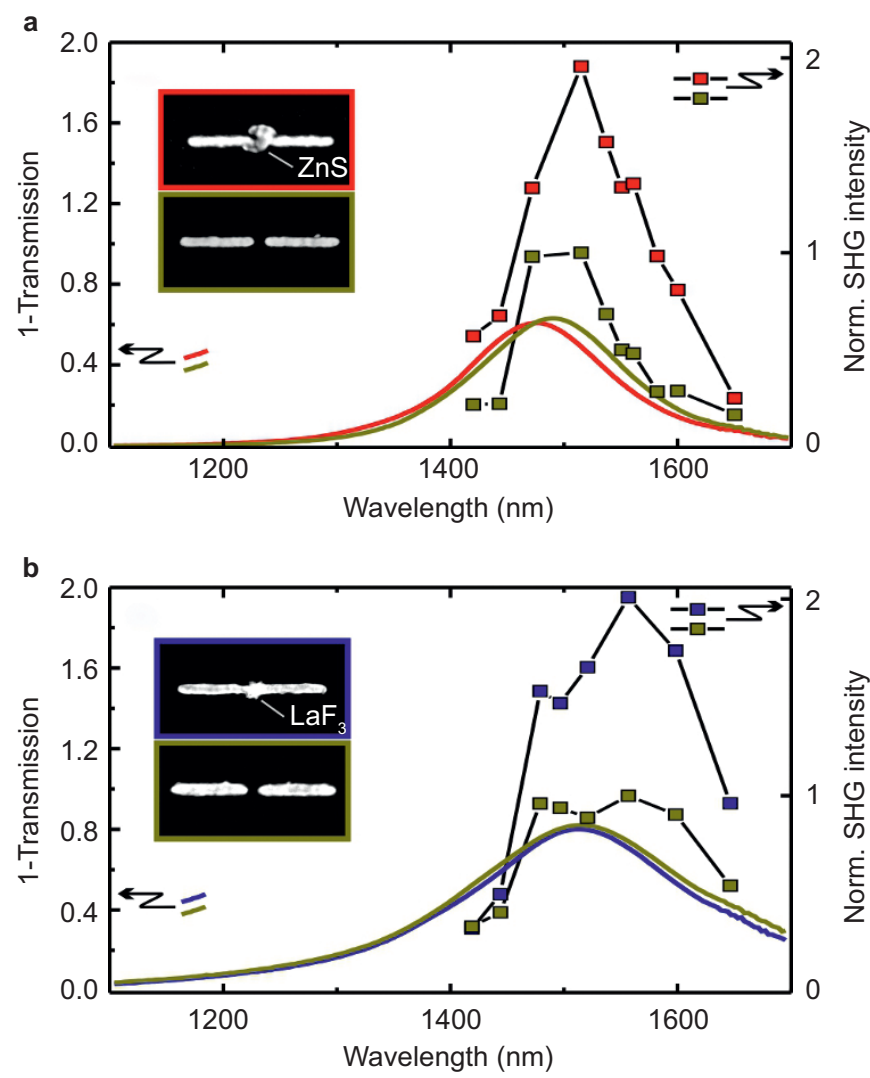

Figure 3 (a) Normal-incidence optical extinction (one minus the measured intensity transmission) spectra for $x$-polarized light (left axis, continuous lines) as well as the SHG intensity (right axis, connected data points) for an array of bare gold nanoantennas (dark yellow) and an array of nanoantennas with ZnS nanoparticles (red), both located on sample 1. (b) The same as in (a) but for an array of bare gold nanoantennas (dark yellow) and an array of nanoantennas with $\mathrm{LaF}_{3}$ nanoparticles (blue), both located on sample 2. The SHG intensity spectra are both cases normalized to the maxima of the corresponding bare gold nanoantenna arrays.

To quantitatively test this qualitative reasoning and to rule out any experimental artifacts, we have performed numerical calculations using the discontinuous Galerkin time-domain method ${ }^{42,43}$ for the bare gold nanoantenna arrays and nanoantenna arrays with a linear dielectric. Our approach self-consistently takes the light propagation at the $\mathrm{SH}$ frequency into account and therefore includes SHG reabsorption, emission shaping and the near-to far-field transition. We described the optical response of the metal by the state-of-the-art hydroynamic Maxwell-Vlasov theory ${ }^{44,45}$, which takes both surface and bulk contributions into account. Its linear limit corresponds to the Drude free-electron model, for which we have chosen a plasma frequency $\omega_{\mathrm{pl}}=1.33 \times 10^{16} \mathrm{rad} \mathrm{s}^{-1}$, a collision frequency $\omega_{\mathrm{col}}=8 \times$ $10^{13} \mathrm{rad} \mathrm{s}^{-1}$, and a background dielectric constant $\varepsilon_{\infty}=9.84$. The refractive index of the glass substrate was taken as $n=1.46$. The geometric parameters of the nanoantennas were adapted from the SEM micrographs shown in Figure 1c and 1d, respectively. The length of the nanoantennas was $350 \mathrm{~nm}$ per arm in the case of the bare gold nanoantennas and $325 \mathrm{~nm}$ in the case of the hybrid nanoantenna. The width and height were approximately $40 \mathrm{~nm}$. The gap had a width of $60 \mathrm{~nm}$ and the size of the dielectric nanoparticles was chosen such that they scarcely touched the nanoantenna arms. The nanoantennas as well as the dielectric nanoparticles were modeled with a surface roughness of approximately $3 \mathrm{~nm}$ root mean square by a random 
displacement of the vertices defining the surfaces of the geometries in the used meshes. This mimicked the experimental conditions and resulted in the observed significant SHG signal. The refractive index of the dielectric nanoparticles was varied to obtain overlapping resonances between the hybrid and bare gold nanoantennas, resulting in a refractive index of $n=3$. To verify the influence of a change of the dielectric environment of the nanoantennas on their SHG efficiency, the second-order nonlinear coefficient of the dielectric nanoparticles was set equal to zero. The linear extinction calculations, as well as the nonlinear SHG simulations, are presented in Figure 4 in the same way as before the experimental results. Both the enhancement of SHG efficiency and the slight narrowing of the resonance line width of the hybrid nanoantennas relative to the bare gold nanoantennas are nicely reproduced. The slight quantitative deviation between the SHG enhancement in the case of the calculation and the experiment can be attributed to experimental as well as numerical uncertainties. The numerical calculations have been repeated, assuming the dielectric nanoparticle to possess an isotropic second-order susceptibility of 2 $\mathrm{pm} \mathrm{V}^{-1}$, which mimics the effective value for a polycrystalline $\mathrm{ZnS}$ nanoparticle. The results are identical to those assuming no nonlinearity for the dielectric nanoparticle. Overall, the numerical results strongly support the above qualitative reasoning in that the SHG signal is strongly influenced by a modification of the dielectric environment.

Subsequently, we studied double-resonant nanoantenna systems consisting of two gap nanoantennas-oriented perpendicular to each other (see Figure la and 1f). One nanoantenna was designed to be resonant to the pump field (long horizontal-orientated nanoantenna), whereas the other nanoantenna was designed to be resonant to the generated SH light (short vertical-orientated nanoantenna). We performed linear extinction and SHG spectroscopy measurements on an array of double-resonant nanoantenna systems with ZnS nanoparticles. For reference, we used an array of only pump-resonant nanoantennas with $\mathrm{ZnS}$ nanoparticles (see Figure 5). Both the pump-resonant nanoantennas and the long nanoantennas of the double-resonant nanoantenna systems had nominally the same length. In the case of the double-resonant nanoantenna systems, we observed a resonance in the extinction spectrum at approximately $780 \mathrm{~nm}$ wavelength for $y$ polarized incident light due to the presence of the short nanoantennas, which was absent in the case of the only pump-resonant nanoantennas. The resonance of the long nanoantennas, which was only visible

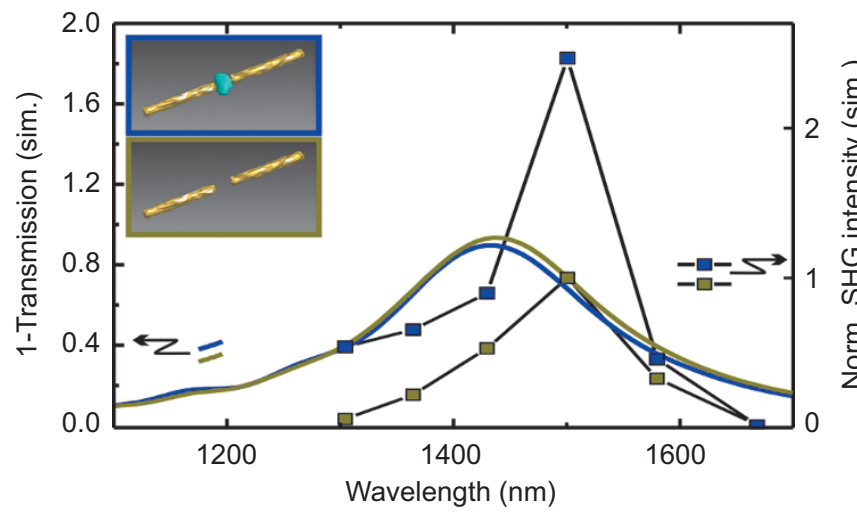

Figure 4 Calculated normal-incidence optical extinction (one minus the calculated intensity transmission) spectra for $x$-polarized light (left axis, continuous lines), as well as the calculated SHG intensity (right axis, connected data points) for an array of bare gold nanoantennas (dark yellow) and an array of nanoantennas with dielectric nanoparticles (blue). The SHG intensity spectra are normalized to the maxima of the corresponding bare gold nanoantenna arrays.

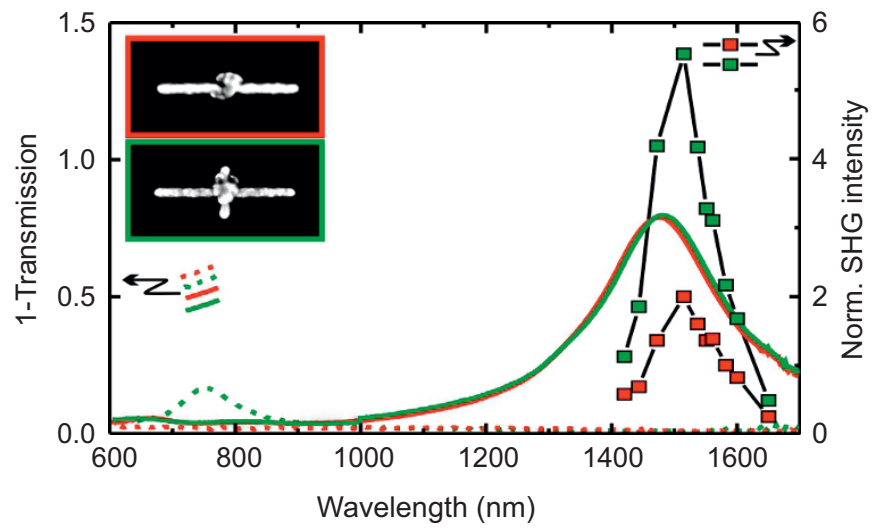

Figure 5 Normal-incidence optical extinction (one minus the measured intensity transmission) spectra for $x$-polarization (left axis, continuous lines) and $y$-polarization (left axis, dashed lines), and the SHG intensity (right axis, connected data points) for an array of ZnS nanoantennas with ZnS nanoparticles (red) and an array of double-resonant nanoantenna systems with ZnS nanoparticles (green). The SHG intensity spectra are both normalized to the maxima of the corresponding bare gold nanoantenna array (see Figure 3a).

for $x$-polarized incident light, was slightly red-shifted, and its line width was marginally increased in the case of the double-resonant systems compared to the only pump-resonant nanoantennas. The red-shift and line width increase originated from a coupling between the short and long nanoantennas. Investigation of the linear extinction spectra of several different double-resonant nanoantenna systems revealed the red-shift and line width increase to be independent of the actual nanoantenna lengths (not shown). By comparing the SHG spectroscopy data of both structures (see Figure 5), we see that the feedback provided by the additional short nanoantennas strongly enhances the SHG efficiency of the hybrid nanoantenna systems, even though the previously mentioned increase of the line width should act counterproductively ${ }^{25,41}$.

The additional short nanoantenna in the case of the double-resonant nanoantenna systems does not only enhance the SHG efficiency but also mediates the coupling to the far field of the generated SH light. This becomes evident upon examination of the polarization properties of the SH light generated by the only pump-resonant nanoantennas with $\mathrm{ZnS}$ nanoparticles and the double-resonant nanoantenna systems with $\mathrm{ZnS}$ nanoparticles. The results are shown in the polar diagrams of Figure 6. In the case of the only pump-resonant nanoantennas, the $\mathrm{SH}$ light was weakly polarized along the long nanoantenna axis ( $x$-polarized), whereas we observed a significant larger degree of polarization in the case of the double-resonant nanoantenna systems, this time along the axis of the short nanoantennas ( $y$-polarized). Our results indicate that double-resonant nanoantenna systems offer the opportunity to influence the far-field properties of the generated SH light.

For the sake of completeness, we checked the SHG efficiency of both nanoantenna geometries for $y$-polarized pump light. However, in both cases, we could not measure any SHG signal within our measurement resolution.

\section{CONCLUSION}

In this work, we compared the SHG efficiency of nonlinear dielectric nanoparticles with plasmonic nanoantennas as well as hybrid dielectric/plasmonic nanoantennas containing either linear or nonlinear dielectric nanoparticles. By combining the results of linear extinction measurements and SHG spectroscopy, we can conclude that an increase of the SHG efficiency of plasmonic nanoantennas obtained 

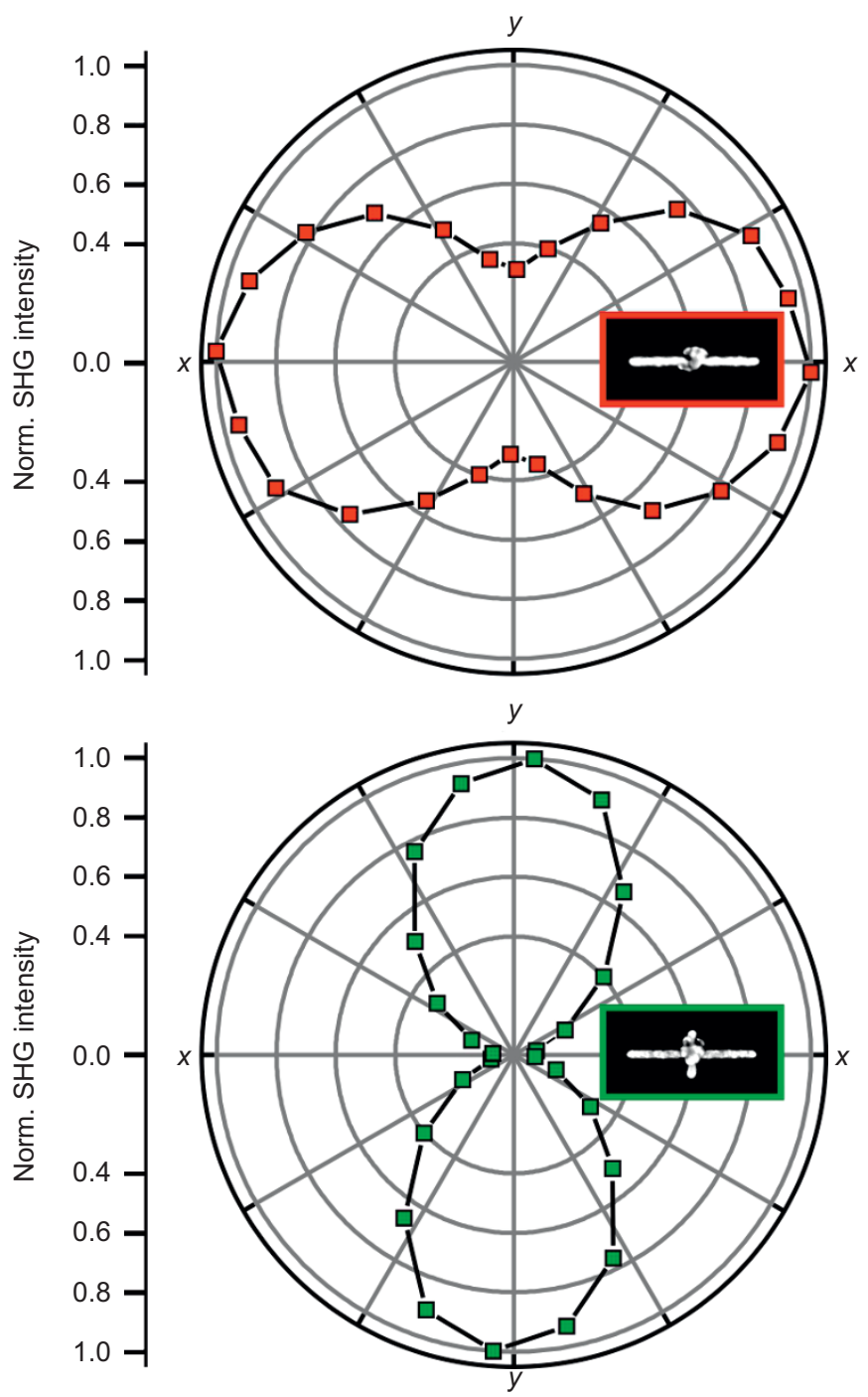

Figure 6 Polar diagrams of the polarization state of the generated SH light from an array of nanoantennas with ZnS nanoparticles (upper diagram) and an array of double-resonant nanoantenna systems with ZnS nanoparticles (lower diagram). In both cases, the pump light was $x$-polarized. All data points are normalized to the maxima of the corresponding data set.

by filling their feed gaps with a dielectric nanoparticle is independent of the nonlinear properties of the dielectric. Furthermore, our experiments showed that the SHG efficiency of plasmonic nanoantennas is several orders of magnitude higher than that of the nonlinear dielectric nanoparticles. The situation might be different if nonlinear dielectrics possessing higher second-order nonlinear coefficients or monocrystalline nonlinear dielectrics with their crystal axis aligned to the local field distributions could be employed.

We additionally showed that a simple combination of two nanoantennas to form a double-resonant nanoantenna system provides not only a strong enhancement of the SHG efficiency but also offers control over the polarization properties of the generated SH signal. In the light of these results, one could also consider the directional emission of the generated $\mathrm{SH}$ light mediated by the second nanoantenna. Because the resonances of this system are highly tunable, this design could be easily adapted for a whole variety of frequency conversion processes.

\section{COMPETING INTERESTS}

The authors declare no competing financial interests.

\section{ACKNOWLEDGEMENTS}

We acknowledge financial support from the Deutsche Forschungsgemeinschaft (SPP1391 and SFB TRR 142). We thank Peter Holik of the Max-Planck Associated Research Center caesar for his support in the sample preparation. The authors gratefully acknowledge the computing time granted by the Paderborn Center for Parallel Computing (PC2).

Hertz H. Ueber sehr schnelle electrische Schwingungen. Ann der Phys 1887; 267: 421-448.

2 Guglielmo Marconi, Ferdinand Braun. Nobel Lecture: Wireless Telegraphic Communication. http://www.nobelprize.org/nobel_prizes/physics/laureates/1909/ marconi-lecture.html, accessed on 10 March 2015.

3 Volakis JL. Antenna Engineering Handbook. 4th ed. New York: McGraw-Hill Professional, Access Engineering, 2007.

4 Bharadwaj P, Deutsch B, Novotny L. Optical antennas. Adv Opt Photon 2009; 1: 438 483.

5 Chen PY, Argyropoulos C, Alù A. Enhanced nonlinearities using plasmonic nanoantennas. Nanophotonics 2012; 1: 221-233.

6 Biagioni P, Huang JS, Hecht B. Nanoantennas for visible and infrared radiation. Rep Prog Phys 2012; 75: 024402.

7 Hasan SB, Lederer F, Rockstuhl C. Nonlinear plasmonic antennas. Mater Today 2014;17:478-485.

8 Boyd RW. Nonlinear Optics. 3rd ed. Burlington, MA: Academic Press, 2008.

9 Fejer MM. Nonlinear optical frequency conversion. Phys Today 1994; 47: 25-32.

10 Petrov V, Ghotbi M, Kokabee O, Esteban-Martin A, Noack F et al. Femtosecond nonlinear frequency conversion based on $\mathrm{BiB}_{3} \mathrm{O}_{6}$. Laser Photon Rev 2010; 4 53-98.

11 Sowade R, Kießling J, Breunig I. CW laser light tunable from blue to red: OPOs pave the way. Photonik Int 2013; 1: 63-65.

12 Nakayama Y, Pauzauskie PJ, Radenovic A, Onorato RM, Saykally RJ et al. Tunable nanowire nonlinear optical probe. Nature 2007; 447: 1098-1101.

13 Zielinski M, Oron D, Chauvat D, Zyss J. Second-harmonic generation from a single core/shell quantum dot. Small 2009; 5: 2835-2840.

14 Vance FW, Lemon BI, Hupp JT. Enormous hyper-Rayleigh scattering from nanocrystalline gold particle suspensions. J Phys Chem B 1998; 102: 1009110093.

15 Bonacina L, Mugnier Y, Courvoisier F, Le Dantec R, Extermann J et al. Polar Fe( $\left(\mathrm{O}_{3}\right)_{3}$ nanocrystals as local probes for nonlinear microscopy. Appl Phys B 2007; 87: 399403.

16 Hsieh CL, Grange R, Pu Y, Psaltis D. Three-dimensional harmonic holographic microcopy using nanoparticles as probes for cell imaging. Opt Express 2009; 17: 2880-2891.

17 Le Xuan L, Brasselet S, Treussart F, Roch JF, Marquier F et al. Balanced homodyne detection of second-harmonic generation from isolated subwavelength emitters. Appl Phys Lett 2006; 89: 121118.

18 Djurišić AB, Leung YH. Optical properties of $\mathrm{ZnO}$ nanostructures. Small 2006; 2 944-961.

19 Danckwerts M, Novotny L. Optical frequency mixing at coupled gold nanoparticles. Phys Rev Lett 2007; 98: 026104.

20 Staedler D, Magouroux T, Hadji R, Joulaud C, Extermann J et al. Harmonic nanocrystals for biolabeling: a survey of optical properties and biocompatibility. ACS Nano 2012; 6: 2542-2549.

$21 \mathrm{Pu}$ Y, Grange R, Hsieh CL, Psaltis D. Nonlinear optical properties of core-shell nanocavities for enhanced second-harmonic generation. Phys Rev Lett 2010; 104: 207402.

22 Jais PM, von Bilderling C, Bragas AV. Plasmon-enhanced second harmonic generation in semiconductor quantum dots close to metal nanoparticles. Papers Phys 2011; $\mathbf{3}$ : 030002.

23 Grinblat G, Rahmani M, Cortés E, Caldarola M, Comedi D et al. High-efficiency second harmonic generation from a single hybrid $\mathrm{ZnO}$ nanowire/au plasmonic nano-oligomer. Nano Lett 2014; 14: 6660-6665.

24 Aouani H, Rahmani M, Navarro-Cía M, Maier SA. Third-harmonic-upconversion enhancement from a single semiconductor nanoparticle coupled to a plasmonic antenna. Nat Nanotechnol 2014; 9: 290-294.

25 Metzger B, Hentschel M, Schumacher T, Lippitz M, Ye X et al. Doubling the efficiency of third harmonic generation by positioning ITO nanocrystals into the hot-spot of plasmonic gap-antennas. Nano Lett 2014; 14: 2867-2872.

26 Thyagarajan K, Rivier S, Lovera A, Martin OJ. Enhanced second-harmonic generation from double resonant plasmonic antennae. Opt Express 2012; 20: 12860-12865.

27 Harutyunyan H, Volpe G, Quidant R, Novotny L. Enhancing the nonlinear optical response using multifrequency gold-nanowire antennas. Phys Rev Lett 2012; 108 : 217403.

28 Chettiar UK, Engheta N. Optical frequency mixing through nanoantenna enhanced difference frequency generation: metatronic mixer. Phys Rev B 2012; 86: 075405. 
29 Ashkin A, Boyd GD, Dziedzic JM. Resonant optical second harmonic generation and mixing. IEEE J Quantum Electron 1966; QE 2: 109-124.

30 Kozlovsky W, Nabors C, Byer R. Efficient second harmonic generation of a diode-laserpumped CW Nd:YAG laser using monolithic $\mathrm{MgO}: \mathrm{LiNbO}_{3}$ external resonant cavities. IEEE J Quantum Electron 1988; 24: 913-919.

31 Zimmermann C, Kallenbach R, Hänsch T, Sandberg J. Doubly-resonant secondharmonic generation in beta-barium-borate. Opt Comm 1989; 71: 229-234.

32 Fiedler K, Schiller S, Paschotta R, Kürz P, Mlynek J. Highly efficient frequencydoubling with a doubly resonant monolithic total-internal-reflection ring-resonator. Opt Lett 1993; 18: 1786-1788.

33 Jin R, Jureller JE, Kim HY, Scherer NF. Correlating second harmonic optical responses of single Ag nanoparticles with morphology. J Am Chem Soc 2005; 127: 12482-12483.

34 Butet J, Duboisset J, Bachelier G, Russier-Antoine I, Benichou E et al. Optical second harmonic generation of single metallic nanoparticles embedded in a homogeneous medium. Nano Lett 2010; 10: 1717-1721.

35 Accanto N, Piatkowski L, Renger J, van Hulst NF. Capturing the optical phase response of nanoantennas by coherent second-harmonic microscopy. Nano Let 2014; 14: 4078-4082.

36 Linnenbank $\mathrm{H}$, Linden $\mathrm{S}$. High repetition rate femtosecond double pass optical parametric generator with more than $2 \mathrm{~W}$ tunable output in the NIR. Opt Express 2014; 22: 18072-18077.

37 Baudrier-Raybaut M, Haïdar R, Kupecek P, Lemasson P, Rosencher E. Random quasiphase-matching in bulk polycrystalline isotropic nonlinear materials. Nature 2004; 432: $374-376$

38 Skipetrov SE. Nonlinear optics: disorder is the new order. Nature 2004; 432 285-286.
39 Morozov E, Kaminskii A, Chirkin A, Yusupov D. Second optical harmonic generation in nonlinear crystals with a disordered domain structure. J Exp Theor Phys 2001; 73: 647-650.

40 Vidal X, Martorell J. Generation of light in media with a random distribution of nonlinear domains. Phys Rev Lett 2006; 97: 013902.

41 Linden S, Niesler FBP, Förstner J, Grynko Y, Meier T et al. Collective effects in secondharmonic generation from split-ring-resonator arrays. Phys Rev Lett 2012; 109: 015502.

42 Hesthaven JS, Warburton T. Nodal Discontinuous Galerkin Methods: Algorithms, Analysis, and Applications. New York: Springer, 2008.

43 Stannigel K, König M, Niegemann J, Busch K. Discontinuous Galerkin time-domain computations of metallic nanostructures. Opt Express 2009; 17:14934-14947.

44 Akhiezer A. Plasma Electrodynamics: Nonlinear Theory and Fluctuations, Vol. 2. New York: Pergamon Press, 1975.

45 Zeng Y, Hoyer W, Liu J, Koch SW, Moloney JV. Classical theory for second-harmonic generation from metallic nanoparticles. Phys Rev B 2009; 79: 235109.

\section{(c) (i) (2) This work is licensed under a Creative Commons Attribution-}

BY NC SA NonCommercial-ShareAlike 4.0 Unported License. The images or other third party material in this article are included in the article's Creative Commons license, unless indicated otherwise in the credit line; if the material is not included under the Creative Commons license, users will need to obtain permission from the license holder to reproduce the material. To view a copy of this license, visit http://creativecommons.org/licenses/by-nc-sa/4.0/ 